\title{
Critical currents in quantum Hall conductors with antidot arrays
}

\author{
G. Nachtwei, Z. H. Liu, G. Lütjering, R. R. Gerhardts, D. Weiss, ${ }^{*}$ K. v. Klitzing, and K. Eberl \\ Max-Planck-Institut für Festkörperforschung, Heisenbergstrasse 1, D-70569 Stuttgart, Germany
}

(Received 29 July 1997; revised manuscript received 3 October 1997)

\begin{abstract}
We have investigated the abrupt onset of dissipation in the quantum Hall effect (QHE) in antidot arrays patterned on a two-dimensional electron system. Different lateral configurations (periodic or aperiodic antidot arrays, and single lines of antidots, with diameters between 40 and $100 \mathrm{~nm}$ and periods or average spacings between 300 and $1500 \mathrm{~nm}$ ) show remarkable differences in their non-Ohmic transport properties. In periodic arrays with large antidot diameter (lithographic diameter $100 \mathrm{~nm}$ ), the breakdown current is systematically reduced with increasing antidot density and determined by the peak value of the local current density. From these measurements, we determined the depletion width around the antidots. In aperiodic arrays, the breakdown current is markedly lower than in periodic arrays of the same antidot size and density due to higher local values of the current density at the same total current. This was experimentally confirmed by measurements of the current dependence of the electron temperature in periodic and aperiodic arrays. Single lines of antidots, placed across the direction of current flow, cause only a small reduction of the breakdown current in comparison with unpatterned reference areas. This is in accordance with the picture of avalanche electron heating for the breakdown of the QHE, where the electrons reach a quasistationary, elevated temperature only after travel distances of several $10 \mu \mathrm{m}$ in a supercritical electric Hall field. In antidot lattices with very small antidot diameter $(40 \mathrm{~nm})$ and small lattice period $(300 \mathrm{~nm})$, the antidots provide additional inelastic scattering, which effectively suppresses the electron heating. This effect overcompensates the geometrical effect of the antidots and was experimentally verified by the observation of higher breakdown currents compared to the reference area. A complete absence of a hot-electron-induced hysteresis in the current-voltage characteristics was observed for this type of array. [S0163-1829(98)06316-4]
\end{abstract}

\section{INTRODUCTION}

The formation of quantum Hall $(\mathrm{QH})$ plateaus at precisely defined values of the Hall resistance ${ }^{1} \rho_{x y}=h / i e^{2}(h$ is Planck's constant, $e$ is electron charge, $i$ is the number of occupied Landau levels) has been used since 1990 to realize the unit of the electric resistance. For high precision measurements, the sample current should be as high as possible but below the critical current where the nearly nondissipative current flow breaks down. Therefore, a lot of experiments on samples with different material properties ${ }^{2-4}$ and geometries $^{5-7}$ have been performed to understand the physics of the breakdown of the QHE. Since these results are still hardly reconcilable within one conclusive model, different mechanisms for the breakdown as intra- (Ref. 8) and interLandau-level transitions, ${ }^{9}$ or a phenomenological description on the basis of electron heating, ${ }^{10,11}$ have been discussed. The breakdown current scales linearly with the sample width for lower electron mobilities. ${ }^{2,3}$ Hence, a nearly homogeneous current distribution exists at currents close to the breakdown in the presence of a high degree of disorder. In contrast, a sublinear increase of the breakdown current with the sample width was observed in high mobility samples, ${ }^{4}$ indicating an inhomogeneous current flow at the breakdown. It was shown experimentally that an inhomogeneous current flow leads to a breakdown in distinct local areas of the sample. ${ }^{5-7}$ Thus, the degree of disorder and the homogeneity of the current distribution are important for the breakdown of the QHE.

We investigate the influence of various lateral antidot arrays (periodic square lattices, aperiodic arrays, and single lines of periodically spaced antidots, placed across the direc- tion of current flow) on the breakdown of the QHE. The antidots cause local geometrical constrictions for the current flow and act as artificial repulsive impurities. We show that both the density and the spatial distribution of antidots are essential for the breakdown. By investigating the change of the breakdown current with the antidot spacing in periodic arrays with a rather large lithographic antidot diameter $\left(d_{L}\right.$ $=100 \mathrm{~nm}$ ), we give evidence that the breakdown is determined by the local maximum of current density in the region between adjacent antidots. In aperiodic arrays, we observe a drastic reduction of the critical currents, compared with periodic arrays of the same antidot density. This is due to an inhomogeneous current flow as a result of the irregular spatial variation of the current density. These results emphasize the importance of local properties on a submicrometer scale for the breakdown of the QHE. However, our results are reconcilable with the recently proposed picture of bootstraptype electron heating. ${ }^{12}$ This is because we observed for single lines of antidots, placed across the current flow direction, a markedly smaller reduction of the breakdown current than for the corresponding periodic square lattices. Only the latter provide sufficiently long traveling paths for the electrons through constrictions between antidots to be effectively heated. Further, our measurements of the electron temperature clearly show the relevance of electron heating ${ }^{10-12}$ for the breakdown of the QHE in antidot arrays.

In a periodic array of small antidots $\left(d_{L}=40 \mathrm{~nm}\right)$ with a small lattice period $\left(a_{0}=300 \mathrm{~nm}\right)$, we observed an unexpected increase of the breakdown current exceeding the value of the unpatterned reference region of the sample. Further, a complete absence of a hot-electron-induced hysteresis in the current-voltage characteristics of the array at integer 
filling factors was observed. We attribute this behavior to additional inelastic scattering for small antidot distances, which reduces the electron temperature and overcompensates the effect of geometrical reduction by the antidots.

\section{SAMPLES}

We have investigated several sets of samples, made from three different $\mathrm{GaAs} / \mathrm{Ga}_{x} \mathrm{Al}_{1-x} \mathrm{As}$ wafers. The first set (called $L$ samples below) was patterned as Hall bars (width $w=50 \mu \mathrm{m})$ on a wafer with an electron density $n_{s}=3.0$ $\times 10^{15} \mathrm{~m}^{-2}$ and a Hall mobility $\mu_{H}=164 \mathrm{~T}^{-1}$ (mean free path $l_{\mathrm{mfp}}=14.9 \mu \mathrm{m}$ ). Two arrays of antidots (periodic with the square lattice constant $a_{0}$ and aperiodic with an average nearest-neighbor spacing $\langle a\rangle$ equal to the lattice constant) were written on each sample by electron beam lithography (EBL) and etched by reactive ion etching (RIE). ${ }^{13}$ The lattice constants of the $L$ samples are 1000, 800, 600, and $400 \mathrm{~nm}$. The lithographic antidot diameter $d_{L}$ is $100 \mathrm{~nm}$. The samples of the second set (called $Z$ samples) are Hall bars ( $w$ $=50 \mu \mathrm{m}$ ) with an electron density of $2.2 \times 10^{15} \mathrm{~m}^{-2}$, mobility $\mu_{H}=96 \mathrm{~T}^{-1}$ and $l_{\mathrm{mfp}}=7.5 \mu \mathrm{m}$. Here, square lattices and single lines with antidot spacings from 1500 to $800 \mathrm{~nm}$ were patterned, applying the same procedure as for the $L$ samples. The third wafer, with the parameters $n_{s}=3.1 \times 10^{15} \mathrm{~m}^{-2}$, $\mu_{H}=60 \mathrm{~T}^{-1}, l_{\mathrm{mfp}}=5.5 \mu \mathrm{m}$ (all values at $T=1.3 \mathrm{~K}$ in the dark), was used to pattern a Hall bar (called $W$ sample, $w$ $=100 \mu \mathrm{m}$, same shape as $L$ samples). This sample contains a periodic array with the smallest antidot diameter and lattice period of $d_{L}=40 \mathrm{~nm}$ and $a_{0}=300 \mathrm{~nm}$, and an unpatterned reference region.

On all samples, we performed dc measurements $(0.2 \leqslant I$ $\leqslant 80 \mu \mathrm{A}$ ) of the longitudinal and Hall resistances, $R_{x x}$ and $R_{x y}$, respectively, in the magnetic field range $0 \leqslant B \leqslant 10 \mathrm{~T}$ and at temperatures of $1.3 \leqslant T \leqslant 24 \mathrm{~K}$. Current-voltage characteristics of the longitudinal voltage $V_{x}$ versus the sourcedrain current were taken near integer filling factors.

\section{EXPERIMENTAL RESULTS AND INTERPRETATION}

\section{A. Breakdown of the QHE in antidot arrays with large antidot diameter: Electron heating in dependence on the lateral configuration of antidots}

The first set of $(L)$ samples was designed to study the influence of the lateral distribution of antidots, defining mesoscopic constrictions, on the breakdown of the QHE. Two arrays of antidots (periodic with the lattice constant $a_{0}$ and aperiodic with the same average antidot spacing $\langle a\rangle$ ) were patterned on each $L$ sample. This arrangement permits an immediate comparison of the QHE breakdown in periodic and aperiodic antidot arrays of the same average antidot density [see inset of Fig. 1(a)]. The distribution function of antidots in the aperiodic arrays is of approximately Gaussian shape with a broadening parameter $\sigma_{a}$ of about $\frac{1}{4}\langle a\rangle$ for all samples. The values of the mean free path at zero magnetic field and $T=1.3 \mathrm{~K}$ in the antidot arrays are comparable to the average antidot spacing (see Ref. 13).

From Shubnikov-de Haas ( $\mathrm{SdH})$ curves with the sample current as parameter, we determined the critical current $I_{c}$ [defined on the basis of a criterion of $\rho_{x x} / \rho_{x y}=\cot \left(\Theta_{H}\right)=3$ $\times 10^{-4}$, with $\Theta_{H}$ being the Hall angle, as a measure for the
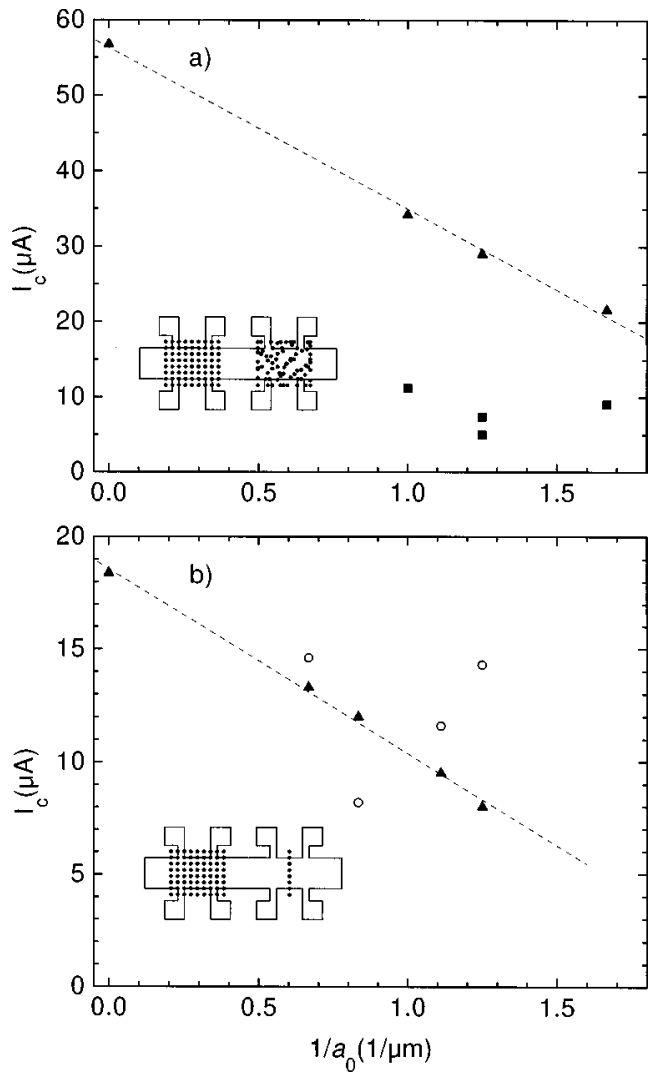

FIG. 1. Critical current $I_{c}(\nu=2)$ vs inverse antidot spacing. (a) for $L$ samples with square lattices $(\boldsymbol{\Lambda})$ and aperiodic arrays $(\boldsymbol{\square})$ of antidots. Inset (a): scheme of the corresponding sample geometry (not to scale). (b) for $Z$ samples with square lattices $(\boldsymbol{\Delta})$ and single lines $(\bigcirc)$ of antidots. Inset (b): sample geometry.

relative dissipation per area]. ${ }^{13}$ For $L$ samples with $a_{0}=\langle a\rangle>400 \mathrm{~nm}$, the critical currents of the periodic arrays are higher than those of the corresponding aperiodic arrays [see Ref. 13 and Eq. (1a)]. For the periodic arrays ( $L$ and $Z$ samples), the critical current $I_{c}$ decreases linearly with increasing $1 / a_{0}$ [Figs. 1(a) and 1(b)]. This linear dependence of $I_{c}$ on $1 / a_{0}$ can be explained by a simple geometrical argument: The breakdown occurs if the highest local current density $j_{x}^{\max }$ between adjacent antidots reaches the critical current density $j_{c}^{0}$ known from the unpatterned reference device:

$$
j_{c}^{0}=j_{x}^{\max }=\frac{I_{c}\left(a_{0}\right)}{w-N \cdot d_{\mathrm{el}}},
$$

leading to

$$
I_{c}\left(a_{0}\right)=I_{c}^{0}\left(1-\frac{d_{\mathrm{el}}}{a_{0}}\right),
$$

where $N=w / a_{0}$ is the number of antidots across the sample, $I_{c}^{0}=j_{c}^{0} w$ is the corresponding critical current, and $d_{\mathrm{el}}$ is the electric antidot diameter. Equation (1) holds for a constant electric Hall field (homogeneous current flow) between the antidot lines (see Appendix). The scaling of $I_{c}$ with $1 / a_{0}$ is equivalent to a linear increase of the critical current with the effective width of the sample with antidots, $N\left(a_{0}-d_{\mathrm{el}}\right)$. This result, obtained on a submicrometer scale, corresponds to the linear dependence of the critical current on the macroscopic 


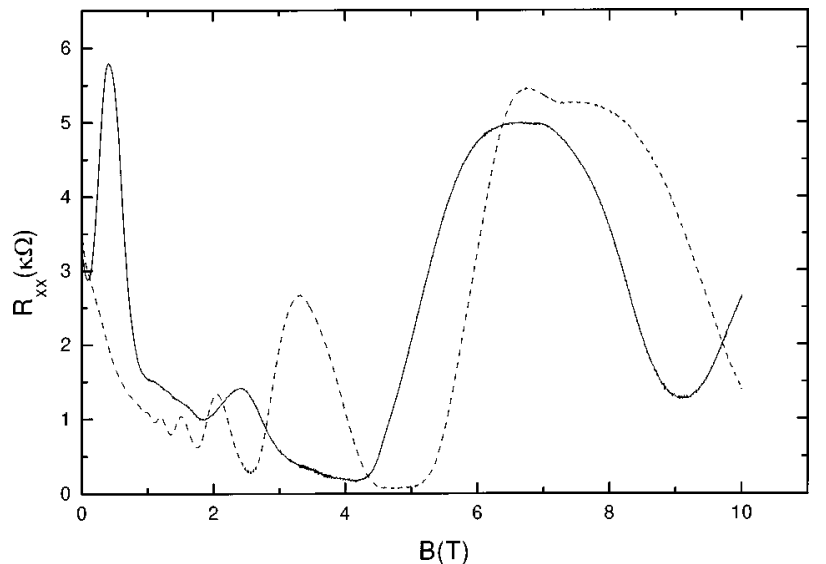

FIG. 2. SdH curves for sample L4a with $\langle a\rangle=a_{0}=400 \mathrm{~nm}(I$ $=1 \mu \mathrm{A}, T=1.3 \mathrm{~K})$. In contrast to the $L$ samples with larger $a_{0}$, the breakdown of the QHE in the aperiodic array (dashed line) is not yet complete, but it is in the periodic one (full line). This is due to $d_{\mathrm{el}} \approx a_{0}$, see text.

sample width as reported earlier. ${ }^{2,3}$ The experimental results agree with the interpretation that the breakdown of the QHE starts where the local current density is at its maximum. The alternative interpretation, that the breakdown would be determined by the current density $\langle j\rangle$ averaged along the current flow direction or the remaining active area of the array, fails to explain the results in a quantitatively correct manner. ${ }^{13}$

From the slope of $I_{c}\left(1 / a_{0}\right)$, values of the electric antidot diameter of $d_{\mathrm{el}}=370$ and $440 \mathrm{~nm}$, and of the critical current density of $j_{c}^{0}=1.1$ and $0.4 \mathrm{~A} / \mathrm{m}$ (for $L$ and $Z$ samples, respectively) can be estimated. The electric antidot diameters are considerably larger than the lithographic ones due to ringshaped depletion layers of about 135 - or 170-nm width ( $L$ or $Z$ samples) around the antidots. As expected, the depletion zones were found larger for smaller carrier densities. The data are consistent with the picture that the current flow occurs exclusively in that region, where an incompressible state of constant local density develops under the conditions of the QHE (Ref. 14) (see Appendix). The value of $d_{\mathrm{el}}=370 \mathrm{~nm}$, obtained for all $L$ samples, is consistent with the transport data obtained in the sample with $a_{0}=\langle a\rangle=400 \mathrm{~nm}$. The QHE is not completely developed in the periodic array with $a_{0}=400 \mathrm{~nm}$ due to a reduction of $a_{0}-d_{\mathrm{el}}$ to values close to zero for $a_{0} \approx d_{\text {el }}$ (see Fig. 2). In contrast, the QHE is still complete in the aperiodic array with $\langle a\rangle=400 \mathrm{~nm}$ (Fig. 2), because the transport current passes preferentially through regions of lower local antidot density than in the corresponding periodic array.

For aperiodic arrays, $I_{c}$ shows no clear dependence on $\langle a\rangle$. The lower critical currents observed in aperiodic arrays can be explained by a strong variation of the local antidot density in the aperiodic arrays as shown by the following argument: if the current is fixed just slightly below the critical value for the periodic array (lattice constant $a_{0}=\langle a\rangle$ ), the breakdown in the corresponding aperiodic array is already complete in all sample regions where $a<\langle a\rangle$ holds. This is due to the local enhancement of the current density between two adjacent antidots for spacings $a<a_{0}=\langle a\rangle$ above the current density of the periodic array (see Appendix). As a consequence, these regions become dissipative $\left(\sigma_{x x}>0\right)$ and will be avoided by the current flow. ${ }^{7}$ The remaining area available for the current flow will therefore be the total array area reduced by the area of all antidots and the areas in between those antidots where $a<\langle a\rangle$ holds. This region contains half of all antidots, but covers less than half of the area of the array. The size of the dissipative region can be calculated using the distribution function, $f(a)$, of the antidots versus their spacing. We have generated a distribution $f(a)$ of the aperiodic patterns that can be well approximated by a Gaussian: ${ }^{13}$

$$
f(a)=\frac{N_{t}}{\sqrt{2 \pi} \sigma_{a}} \exp \left\{-\frac{1}{2}\left(\frac{a-\langle a\rangle}{\sigma_{a}}\right)^{2}\right\},
$$

with $N_{t}$, the total number of antidots, and nearly the same value $\sigma_{a} /\langle a\rangle=0.28$ for all $L$ samples. This yields for the area containing antidots with spacings $a<\langle a\rangle$ :

$$
A(a \leqslant\langle a\rangle)=\int_{0}^{\langle a\rangle} a^{2} f(a) d a \approx 0.32 N_{t}\langle a\rangle^{2},
$$

where $N_{t}\langle a\rangle^{2}$ is the sample area, and the prefactor is independent of $\langle a\rangle$ for $\sigma_{a} /\langle a\rangle=$ constant. The remaining nondissipative area is further reduced by half of the total area of all antidots, $\left(N_{t} / 2\right)(\pi / 4) d_{\mathrm{el}}^{2}$. In contrast, in the periodic array the reduction of area is given by the total area of all antidots, $N_{t}(\pi / 4) d_{\mathrm{el}}^{2}$, only. For $d_{\mathrm{el}} /\langle a\rangle<0.9$ (with $d_{\mathrm{el}}=370 \mathrm{~nm}$, this means for $\langle a\rangle>410 \mathrm{~nm}$ ), the area available for the nondissipative current flow is smaller in the aperiodic array than in the periodic one at a current slightly below the breakdown value of the periodic array. Thus, for the same total current, a typical local current density will be higher in the aperiodic than in the periodic array. Consequently, the breakdown of the QHE will occur at a lower total current in the aperiodic array than in the periodic one. The same argument leads to the opposite result for $d_{\mathrm{el}} /\langle a\rangle>0.9$ (i.e., for our sample with $\langle a\rangle=400 \mathrm{~nm}$ ), due to the finite probability of overlap of the antidots. This overlap gives rise to a larger area remaining for the current flow in the aperiodic array, in accordance with our experimental results obtained on the sample with $a_{0}$ $=\langle a\rangle=400 \mathrm{~nm}$ (Fig. 2).

Due to the percolative nature of the current flow in aperiodic arrays, the local enhancement of the current density, which leads to the breakdown, can be different from sample to sample, even if $\langle a\rangle$ and $\sigma_{a}$ have the same values. This explains the difference of $I_{c}$ found in two aperiodic arrays of the same value for $\langle a\rangle=800 \mathrm{~nm}$.

The correlation of the QHE breakdown with the highest local current density does not contradict the recently proposed nonlocal electron heating approach, ${ }^{12}$ as the antidot array extends over a length of more than $50 \mu \mathrm{m}$. The electrons are subsequently heated while passing the array line by line. This could be confirmed by the results obtained on samples with single antidot lines. Figure 1(b) shows the results, obtained on the $Z$ samples. The breakdown currents of the sample areas containing a single line are usually larger (the exceptional case for $a_{0}=1200 \mathrm{~nm}$ is attributed to a local inhomogeneity of the carrier density in the sample region with the antidot line) than those of the corresponding square lattice. Although a single line causes the same local enhance- 


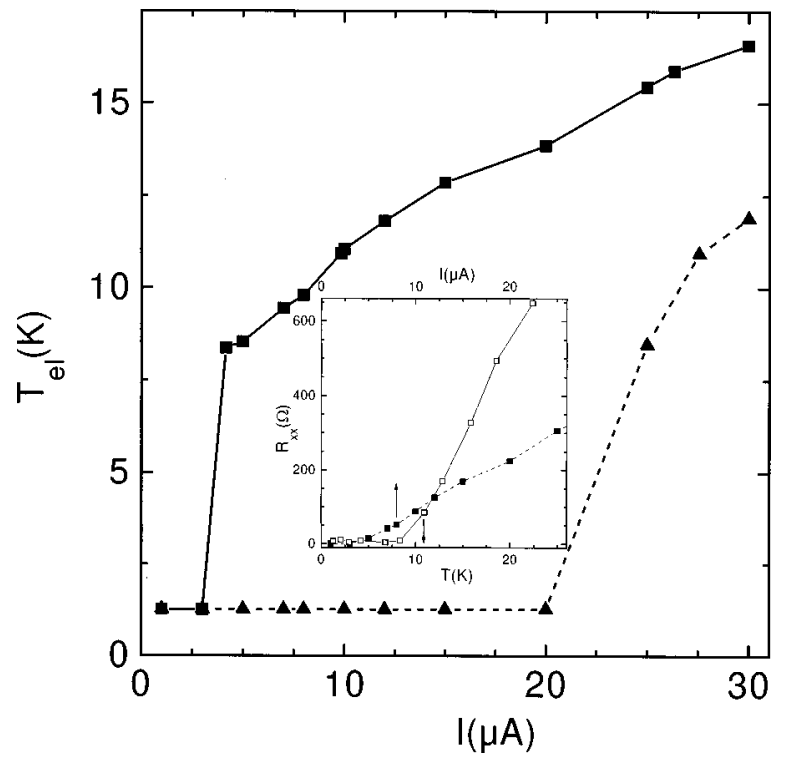

FIG. 3. Temperature $T_{\mathrm{el}}$ of hot electrons versus sample current around the QHE breakdown $(\nu=2)$ in a periodic $(\boldsymbol{\Lambda})$ and the cor-

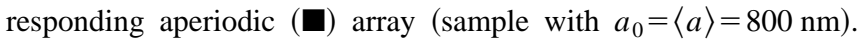
Inset: Longitudinal resistance $R_{x x}$ in dependence on the current (ם) and on the temperature $(\square)$ for the aperiodic array.

ment of the current density as the lattice, the extension of this constriction along the current flow direction of some 100 $\mathrm{nm}$ is obviously far too small to effectively heat the electrons. In the lattice, the traveling distance of the electrons is large enough to develop a stationary state of elevated electron temperature. These results show that the electron heating is governed both by the local enhancement of the current density and by the extension of the current path containing the constrictions. The electron heating model explains the QHE breakdown by the balance of energy gain (per unit area and time) due to the current, $\rho_{x x} j^{2}$, and the corresponding loss, due to the relaxation of the heated electrons:

$$
\rho_{x x}\left(T_{\mathrm{el}}\right) j^{2}=\frac{\varepsilon\left(T_{\mathrm{el}}\right)-\varepsilon\left(T_{L}\right)}{\tau_{e p}},
$$

where $\varepsilon(T)$ is the energy of the electron system (per unit area) at the electron [lattice] temperature $T_{\mathrm{el}}\left[T_{L}\right]$, and $\tau_{e p}$ is the energy relaxation time of the heated electrons. Equation (4) takes the temperature dependence of $\rho_{x x}$ into account in terms of the electron temperature only. ${ }^{10,11}$ Using this assumption, a comparison of the $\rho_{x x}$ values measured at low currents and different lattice temperatures $\left(T_{\mathrm{el}}=T_{L}\right)$ with those measured at low lattice temperature and different currents $\left(T_{\mathrm{el}}>T_{L}\right)$ yields the electron temperature as a function of the current (see inset of Fig. 3). We employed this method to deduce the electron temperatures near the breakdown of the QHE in the center of the QH plateau at $\nu=2.0$. Figure 3 shows a comparison of the electron temperature in the periodic and the aperiodic array of a sample with $a_{0}=\langle a\rangle$ $=800 \mathrm{~nm}$. Near the breakdown current, the electron temperature rises from the lattice temperature of $1.3 \mathrm{~K}$ to values of about $10 \mathrm{~K}$. In the aperiodic array, the electron temperature starts to rise at lower currents than in the periodic array. We attribute this behavior to the higher local current densities in the regions where the current actually flows and to the superlinear increase of energy dissipation with the current density.

The electron temperatures obtained from transport measurements integrate the sample properties over the area of the array and correspond to the steady state of the hot electron avalanche. ${ }^{12}$ Therefore, the measured electron temperatures may differ considerably from local values on a mesoscopic scale. Further, Eq. (4) is valid only for small differences between electron and lattice temperature, since the electronphonon scattering rate increases with this difference. Hence, the values obtained for the electron temperature can only be taken as an estimate, but clearly show the correlation between current-induced breakdown of the QHE and heating of the electron system.

\section{B. Breakdown of the QHE in antidot arrays with small antidot diameter: Electron heating in the presence of additional scattering at the antidot array}

The breakdown behavior described so far was investigated in samples with relatively large antidots $\left(d_{\mathrm{el}}=370-440 \mathrm{~nm}\right)$ and large lattice periods $\left(a_{0}\right.$ $=400-1500 \mathrm{~nm}$ ). All results could be explained by a predominantly geometrical influence of the antidots, due to their large effective diameter. However, we observed a completely different and unexpected breakdown behavior on the $W$ sample with a small antidot diameter $\left[d_{L}=40 \mathrm{~nm}, d_{\mathrm{el}}=100\right.$ $\mathrm{nm}$ (Ref. 15) after illumination] and small lattice period of $a_{0}=300 \mathrm{~nm}$. In contrast to the reduction of the critical current in arrays of large antidots, we observed an enhancement of the critical current above the value of the unpatterned reference region in the array of small antidots (Fig. 4). In this case, a qualitatively different effect of the "small" antidot lattice on the breakdown of the QHE has to be assumed.

Apart from the different critical currents, the $I-V$ characteristics measured on the antidot lattice and on the unpatterned reference region showed very striking differences (Fig. 5). In the unpatterned region, a clear hysteresis between up and down sweep of the current is present for the negative current direction. For forward currents, both the value of breakdown current and the shape of the hysteresis are different from those measured for backward currents. We attribute this to a local variation of the electron temperature, either due to probing of different states of the electron avalanche for different distances between the current injecting and the potential probing contacts, ${ }^{12}$ or to strong inhomogeneities of the current flow in the unpatterned region. In contrast, no hysteresis in the $I-V$ curves was found for the antidot array. Further, $I_{c}$ is approximately the same for both current directions.

In this section, we provide an explanation of the enhancement for the critical current by an increase of the inelastic scattering rate of electrons due to the antidots, leading to a reduced electron heating [Eq. (4)]. We will show that, if the increase of the scattering rate due to the antidot array dominates over the effective reduction of the sample area by the antidots, the critical current of the periodic antidot array can exceed the value of the unpatterned region. This condition was obviously realized for the $W$ sample with small antidots (after illumination), as shown in Fig. 4(b).

To explain our experimental results, we invoke the hotelectron model ${ }^{10,11}$ and calculate the dependence of the elec- 

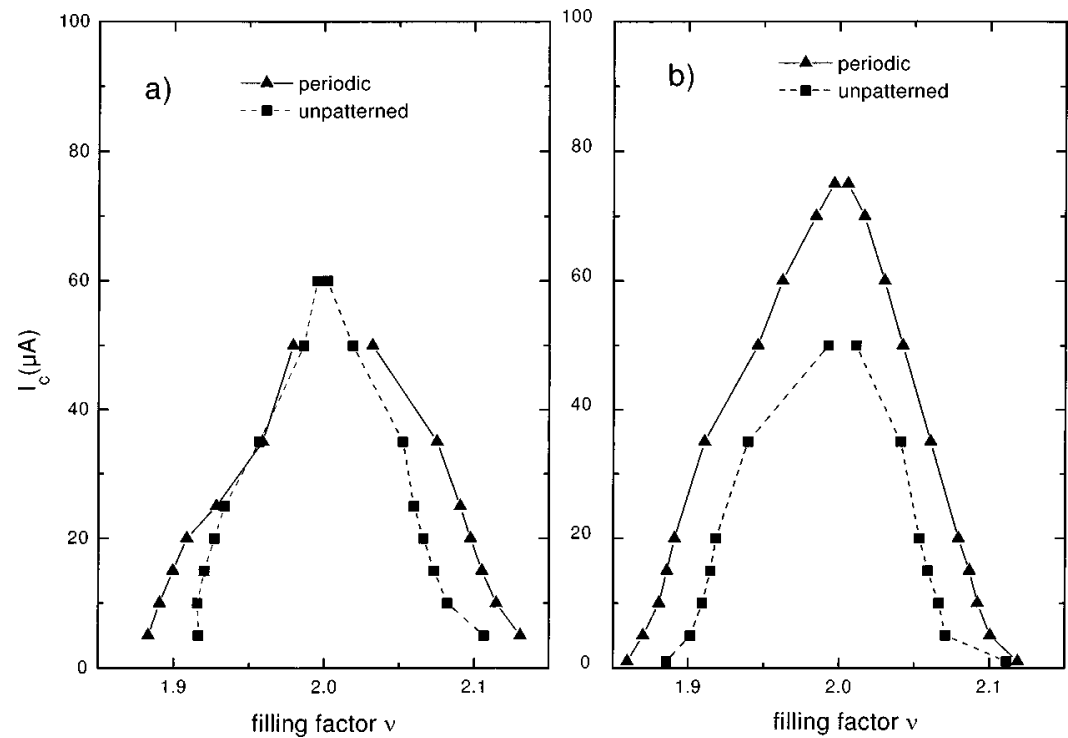

FIG. 4. Critical current vs filling factor for the $W$ sample $(T=1.3 \mathrm{~K})$, (a) sample before and (b) after illumination.

tron temperature $T_{\mathrm{el}}$ on the sample current for the reference sample and the antidot array. The essential difference in electron heating between the unpatterned region and the antidot array is the inelastic scattering rate $1 / \tau$. In unpatterned samples, $\tau$ is supposed to be determined by electron-phonon interaction and can be approximated by the relation ${ }^{11}$

$$
1 / \tau_{e p}=C_{e p} T_{\mathrm{el}}^{2}
$$

with $C_{e p}=(1.2-1.5) \times 10^{7} \mathrm{~K}^{-2} \mathrm{~s}^{-1}$ as empirical constant. The constant $C_{e p}$ is a material parameter for inelastic scattering in GaAs and was experimentally found to be only weakly dependent on the sample properties and the magnetic field. ${ }^{11}$ We therefore adopt the value given in Ref. 11 for our calculations.

For even integer filling factors, the Fermi energy $E_{F}$ is located in the middle of the Landau gap, which is $10.4 \mathrm{meV}$ for the $W$ sample at $B=6.0 \mathrm{~T}(\nu=2.0)$. The thermal energy at the lattice temperature of $T_{L}=1.3 \mathrm{~K}$ is only $0.11 \mathrm{meV}$. Hence, the density of states (DOS) entering $\varepsilon(T)$ can be assumed to be constant $\left(D_{B G}\right)$ near the Fermi energy due to the absence of screening effects. ${ }^{16,17}$ This holds even for elevated electron temperatures, since the temperature corresponding to the energy difference between the Fermi energy and the next higher Landau level is about $60 \mathrm{~K}$. Therefore, the energy of the electron system, $\varepsilon(T)$, can be explicitly given (for $E_{F}$ in the middle of the Landau gap) by

$$
\varepsilon(T)=2 \int_{E_{F}}^{\infty}\left(E-E_{F}\right) D(E) f\left(E, E_{F}\right) d E=\frac{\pi^{2}}{6} D_{B G}(k T)^{2},
$$

with $f\left(E, E_{F}\right)$ being the Fermi-Dirac distribution function. Using Eqs. (4)-(6), the following relation for $j_{x}\left(T_{\text {el }}\right)$ can be obtained for the unpatterned sample region:

$$
j_{x}\left(T_{\mathrm{el}}\right)=\left\{\frac{\pi^{2} k^{2}}{6} D_{B G} C_{e p} \frac{T_{\mathrm{el}}^{4}-T_{\mathrm{el}}^{2} T_{L}^{2}}{\rho_{x x}\left(T_{\mathrm{el}}\right)}\right\}^{1 / 2} .
$$

Equation (7) yields an $\mathrm{S}$-shaped $T_{\mathrm{el}}$ versus $j_{x}$ dependence. Since $\rho_{x x}$ is a monotonous function of $T_{\mathrm{el}}$, this corresponds to an S-shaped $V_{x}-I$ characteristic. ${ }^{10,11}$ As the parts of the curve, where $\partial T_{\mathrm{el}} / \partial j_{x}<0$ (and $\partial V_{x} / \partial j_{x}<0$, respectively) holds, are instable, a hysteresis in the corresponding experimental curve develops. The hysteresis is confined between two limiting values of $j_{x}, j_{c 1}$, and $j_{c 2}\left(j_{c 1}\right.$ corresponds to lower and $j_{c 2}$ to higher electron temperatures, $j_{c 1}>j_{c 2}$ ). Both values, $j_{c 1}$ and $j_{c 2}$, are not only dependent on the parameters of Eq. (7), as $D_{B G}$ and $C_{e p}$, but also on the temperature dependence of $\rho_{x x}\left(T_{\mathrm{el}}\right)$. To explain quantitatively the hysteresis of the $V-I$ characteristics that we observed for the unpatterned region [Fig. 5(a)], we use the ansatz $^{16}$

$$
\rho_{x x}=\rho_{0} \exp \left\{-\Delta / k T_{\mathrm{el}}\right\}+\rho_{B G},
$$

with $\Delta=\hbar \omega_{c} / 2$ for $E_{F}$ in the middle of the Landau gap. The first term in Eq. (8) describes the resistivity contribution due to thermal activation over the Landau gap. Using the tensor relation $\rho_{x x}=\sigma_{x x} /\left(\sigma_{x x}^{2}+\sigma_{x y}^{2}\right)$ and $\sigma_{x x}(T)=\sigma_{0} \exp \{-\Delta / k T\}$, the prefactor $\rho_{0}$ can be determined. For long-range scattering, $\sigma_{0}$ was theoretically found ${ }^{18}$ equal to $2 e^{2} / h$, as confirmed by measurements of Svoboda et al. ${ }^{19}$ At a filling factor of $\nu=2$ with $\sigma_{x y}=2 e^{2} / h$, this leads to $\rho_{0}=h / 4 e^{2}$ (see also Ref. 20). The additional contribution to the resistivity, $\rho_{B G}$, has crucial influence on the breakdown current density $j_{c 1}$. If purely activated behavior of $\rho_{x x}(T)$ is assumed, the electric power gain $\rho_{x x} j_{x}^{2}$ is very small at low temperatures, even for rather large values of $j_{x}$. Thus, the values for $j_{c 1}$ become unrealistically high. To obtain values of $j_{c 1}$ closer to the experiment, finite values of $\rho_{B G}$ have to be assumed. A possible origin of $\rho_{B G}$ is variable range hopping $(\mathrm{VRH}){ }^{21,22}$ Different temperature dependences and parameters for $\rho_{B G}$ were proposed. ${ }^{21,22}$ However, the value of $j_{c 2}$, which marks the lower limit of the hysteresis at higher electron temperatures, is nearly unaffected by the choice of $\rho_{B G}$. For the determination of $j_{c 2}$, the activated conduction provides the dominating contribution. Using the following parameters: (1) The density of states, $D_{B G}=2 \times 10^{9} \mathrm{~cm}^{-2} \mathrm{meV}^{-1}$, as reported in Ref. 16 for comparable samples, (2) the electronphonon scattering constant, $C_{e p}=1.2 \times 10^{7} \mathrm{~K}^{-2} \mathrm{~s}^{-1}$, as re- 

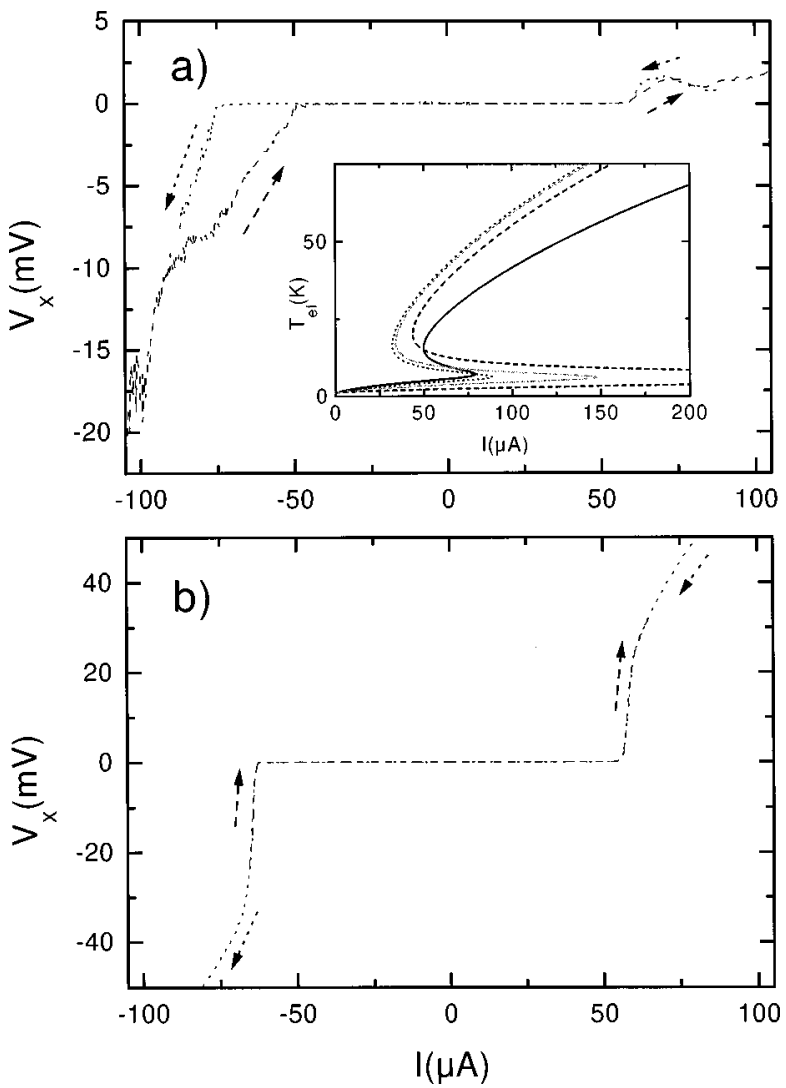

FIG. 5. $V_{x}$ vs sample current for the $W$ sample [(a) reference region, (b) antidot array]. No hysteresis is observable in the antidot array. Inset in (a): Electron temperatures vs current for sample $W$, calculated applying Eqs. (7) and (11), for the normal region (full line) and for the antidot lattice (dashed: scattering probability $p_{a}$ $=1$ and drift length $l_{\text {drift }}=a_{0}=300 \mathrm{~nm}$, dash-dotted: $p_{a}=0.3, l_{\text {drift }}$ $=1 \mu \mathrm{m}$, dotted: $\left.p_{a}=0.17, l_{\mathrm{drift}}=2 \mu \mathrm{m}\right)$.

ported in Ref. 11 for the filling factor $\nu=2$, and (3) the activation energy $\Delta=\hbar \omega_{c} / 2 \propto B$, we obtained the lower limit of the hysteresis $\left(I_{c 2}=j_{c 2} w\right)$ for the $I-V$ characteristics of the unpatterned sample in excellent agreement with the experiment [Fig. 5(a) and inset]. To reproduce the upper limit of the observed hysteresis $\left(I_{c 1}=j_{c 1} w\right)$ by the calculations, we tested several temperature dependences for $\rho_{B G}$. The best fit was obtained for values approaching $\rho_{B G} \approx 1 \Omega$ at electron temperatures near the breakdown. The essential conclusion from this finding is that the breakdown of the QHE can be explained consistently within the hot electron model only, if $\rho_{x x}$ is predominantly determined by additional transport mechanisms (i.e., hopping) in the pre-breakdown regime.

The material parameters $D_{B G}$ and $\rho_{B G}$, which provide the best fit for the $I-V$ characteristics of the unpatterned sample, were also used in the calculations for the antidot array. For the inelastic scattering time, however, we assume that hot electrons can lose energy while interacting with the antidot lattice, due to the local enhancement of the Hall electric field between adjacent antidots (see Appendix). We do not address the question of the microscopic nature of the electronphonon interaction in the antidot array, but presume simply that $\tau$ is determined by the average drift time between two inelastic scattering events in the antidot lattice as long as $\tau_{\text {drift }} \ll \tau_{e p}$ holds. The drift time $\tau_{\text {drift }}$, corresponding to the mean drift length $l_{\text {drift }}$ between two scattering events, can be expressed as follows:

$$
\tau_{\text {drift }}=l_{\text {drift }} / v_{\text {drift }}=a_{0} e n_{s} / j_{x} p_{a}
$$

with $p_{a}=a_{0} / l_{\text {drift }}$ being the probability of inelastic relaxation of an electron while passing one elementary cell of the antidot lattice. The drift velocity $v_{\text {drift }}=j_{x} / e n_{s}$ is relevant in our case, since the magnetic length $l_{B}$ is more than one order of magnitude smaller than the antidot lattice constant $a_{0}$ (drifting-orbit regime ${ }^{15}$ ). At higher electron temperatures beyond the breakdown, the condition $\tau_{\text {drift }} \ll \tau_{e p}$ is violated in the antidot lattice. Then, the total scattering rate $1 / \tau$ can be written as the sum of the rates due to electron-phonon scattering and due to inelastic scattering events in the antidot lattice:

$$
\frac{1}{\tau}=\frac{1}{\tau_{e p}}+\frac{1}{\tau_{\mathrm{drift}}}=C_{e p} T_{\mathrm{el}}^{2}+\frac{j_{x} p_{a}}{a_{0} e n_{s}} .
$$

This total scattering rate yields the following current density as a function of the electron temperature in the antidot array:

$$
j_{x}\left(T_{e l}\right)=\frac{1}{2} j_{\text {array }}+\left(\frac{1}{4} j_{\text {array }}^{2}+j_{\text {ref }}^{2}\right)^{1 / 2}
$$

with

$$
j_{\text {array }}\left(T_{\mathrm{el}}\right)=\frac{\pi^{2} k^{2}}{6 e} \frac{D_{B G} P_{a}}{n_{s} a_{0}} \frac{T_{\mathrm{el}}^{2}-T_{L}^{2}}{\rho_{x x}\left(T_{\mathrm{el}}\right)}
$$

and $j_{\text {ref }}$ according to Eq. (7).

Hence, the electron temperatures in the antidot lattice beyond the breakdown will be limited by the intrinsic electronphonon scattering as in the unpatterned system, and so will be the low-current side $I_{c 2}$ of the $I-V$ hysteresis. The higher electron temperatures and the lower $I_{c 2}$ values in the antidot lattice [in comparison with the unpatterned system, see inset in Fig. 5(a)] are due to the reduction of the effective sample width by $w_{\text {eff }}=w_{0}\left(a_{0}-d_{\mathrm{el}}\right) / a_{0}$ in the antidot array. Further, the hysteresis limits $I_{c 1}$ and $I_{c 2}$ shift to higher values, the closer the distance between the additional scattering events in the antidot lattice is. As visible in the inset of Fig. 5(a), for scattering probabilities $0.17<p_{a} \leqslant 1$ or drift lengths $2 \mu \mathrm{m}$ $>l_{\text {drift }} \geqslant 300 \mathrm{~nm}$, the decrease of the critical current $I_{c 1}$ by the reduction of $w_{\text {eff }}$ is overcompensated by the additional scattering. In particular, high scattering rates (i.e., high probabilities $p_{a}$ and/or small lattice constants $a_{0}$ ) lead to critical currents, which can exceed the value of the unpatterned region considerably. This is the principal explanation for the fact, that an effective enhancement of the critical current could be observed only in the antidot lattice with the smallest antidot lattice period investigated in this study. The complete absence of any hysteresis in the antidot lattice, however, cannot be straightforwardly explained within this picture. A qualitative explanation for the absence of the $I-V$ hysteresis can be given by the obviously higher degree of homogeneity of the current flow in the antidot lattice, compared with the unpatterned region. The formation and spatial fluctuation of local hot-electron domains on a large scale is assumed to be essential for the observation of a hysteresis in the $I-V$ characteristics. ${ }^{11}$ This effect, which implies a spatial decom- 
position of the systems into areas with hot and cold electrons near the breakdown, ${ }^{11}$ could be effectively suppressed in the presence of a homogenizing antidot lattice. This assumption is in accordance with our experimental results. In our experiment, the critical current of the unpatterned region decreases slightly after illumination, although it should increase as observed for the antidot array (see Fig. 4), due to the shift of the $\mathrm{QH}$ plateau to higher magnetic fields. This indicates clearly an increasing degree of inhomogeneity in the local distribution of the electron density for the unpatterned region, whereas a higher degree of homogeneity can be presumed in the antidot lattice, in accordance with the observed increase of $I_{c}$ after illumination.

In conclusion, the hot-electron model ${ }^{10,11}$ explains reasonably well the behavior of the $I-V$ characteristics of the QHE breakdown, observed on unpatterned samples. The complete absence of hysteresis effects in antidot lattices, however, cannot be reproduced straightforwardly by this model. Nevertheless, the model yields an appropriate relation between the breakdown current enhancement, which was observed in our experiments on small-period lattices of small antidots, and the increase of the inelastic scattering rate in these antidot lattices.

\section{SUMMARY}

We have measured the critical currents of the QHE on Hall bars with antidot arrays. In periodic arrays with rather large antidots $\left(d_{L}=100 \mathrm{~nm}, d_{\mathrm{el}}=370-440 \mathrm{~nm}\right)$, the breakdown is governed by the increase of the maximum local current density between neighboring antidots. The critical current is reduced with decreasing distance between the antidots due to the corresponding increase of the current density between the antidots, which are surrounded by rather large depletion zones. For aperiodic arrays, the breakdown current is strongly reduced in comparison with periodic arrays of the same antidot density, due to the inhomogeneous distribution of the antidots, which leads to a strongly inhomogeneous current flow. This is confirmed by measurements of the electron temperature, which in an aperiodic array rises at lower currents and to higher values than in the corresponding periodic array.

Single lines of antidots, placed across the current flow direction, only slightly reduce the breakdown current. This confirms the assumption of avalanche heating of electrons. The length of the constriction formed by a single line along the current flow direction, which is of the order of some 100 $\mathrm{nm}$ only, is not sufficient to reach the stationary state of the hot-electron avalanche.

In samples with small antidot diameters and periods, the effect of the geometrical reduction can be compensated for by an effective reduction of the electron temperature, if a sufficient increase of the inelastic scattering rate due to the antidot lattice occurs. Our observation of considerably higher breakdown currents of the antidot lattice $\left(a_{0}=300 \mathrm{~nm}\right.$ and $d_{L}=40 \mathrm{~nm}$ ) in comparison with the unpatterned sample region is, however, partially due to the higher degree of homogeneity in the current flow through the antidot lattice. This explains also the observed absence of any hysteresis in the current-voltage characteristics of antidot lattices, in contrast to the behavior of unpatterned samples.

\section{ACKNOWLEDGMENTS}

We thank W. Dietsche, D. Pfannkuche, and B. Farid for valuable discussions. The wafers $L$ and $W$ investigated in this study were grown by C. T. Foxon at Philips Research Laboratories, Redhill Surrey RH1 5HA, UK, and by G. Weimann, FTZ der Bundespost, P.O. Box 5000, 64295 Darmstadt, Germany.

\section{APPENDIX}

Our understanding of the different breakdown currents in periodic and aperiodic arrays of antidots is based on the assumption that, in general, the average current density between adjacent antidots increases with decreasing distance between these antidots. The purpose of this Appendix is to precisely formulate and to check this assumption for a welldefined typical situation.

We consider a square lattice of lattice constant $a$, with two antidots per unit cell, located at $\mathbf{r}_{0}=\left(0, y_{0}\right)$ and $-\mathbf{r}_{0}$. Further, we assume a stationary current flow through the lattice, with an average current density $\mathbf{j}^{0}=\langle\mathbf{j}(\mathbf{r})\rangle=\left(j_{x}^{0}, 0\right)$ in the $x$ direction. Then the electric field can be derived from an electrostatic potential, $\mathbf{E}=-\nabla \Phi$, and the equation of continuity requires $\boldsymbol{\nabla} \cdot \mathbf{j}=0$. We apply a local Drude relation between current density and field, $\mathbf{j}(\mathbf{r})=\boldsymbol{\sigma}(\mathbf{r}) \mathbf{E}(\mathbf{r})$, with $\sigma_{x x}$ $=\sigma_{y y}=\sigma_{l}(\mathbf{r})$ and $\sigma_{y x}=-\sigma_{x y}=\sigma_{H}(\mathbf{r})$. To describe the effect of an antidot at the position $r_{0}$, we assume a reduction of the electron density by the factor $\{1-\exp (-[r$ $\left.\left.\left.-r_{0}\right]^{2} / R^{2}\right)\right\}$. Thus, the electron density is reduced to zero at the center $\mathbf{r}_{0}$ of an antidot, but is practically unaffected by that antidot at a distance $\left|\mathbf{r}-\mathbf{r}_{0}\right|>2 R$. Positions and radii of the antidots are chosen such that there is no overlap of antidots belonging to different unit cells of the lattice $\left(a / 2-y_{0}\right.$ $>2 R$ ). In the spirit of the Drude theory, we presume that the local value of the conductivity is proportional to the local electron density, $\sigma_{l}(\mathbf{r}) / \sigma_{l}^{h}=\sigma_{H}(\mathbf{r}) / \sigma_{H}^{h}=n_{s}(\mathbf{r}) / n_{s}^{h}$, where $\sigma_{l}^{h}, \sigma_{H}^{h}$, and $n_{s}^{h}$ are longitudinal conductivity, Hall conductivity, and electron density of the homogeneous electron system without the antidots. To adapt this model to the quantum Hall effect near to its breakdown, we take into account that $\sigma_{l}^{h}$ is finite, but very much smaller than $\sigma_{H}^{h}$. Applying these model assumptions, we obtain the second-order partial differential equation

$$
-\boldsymbol{\nabla} \cdot[\boldsymbol{\sigma}(\mathbf{r}) \boldsymbol{\nabla} \Phi(\mathbf{r})]=0
$$

for the electrostatic potential, which we solve numerically by Fourier transformation. Figure 6 shows the equipotential lines obtained for the parameter values $R / a=0.05, y_{0} / a$ $=0.15$ (aperiodic array), and $\sigma_{l}^{h} / \sigma_{H}^{h}=10^{-3}$. Since the Hall angle is very close to $90^{\circ}$, the current flows practically along the equipotential lines. Apparently, the current is completely expelled from the interior of the antidots, where the density is reduced from the value $n_{s}^{h}$ by more than a few percent, and where the potential is constant. Figure 7(a) shows the corresponding result for the $x$ component of the current density $j_{x}(x, y)$ along the lines $x=0$ through the center of the antidots and $x / a=0.5$ midway between two adjacent lines of antidots. The current density at $x / a=0.5$ deviates only weakly from the corresponding value $j_{x}^{0}$ of the homogeneous system without antidots. At $x=0$, however, the current den- 


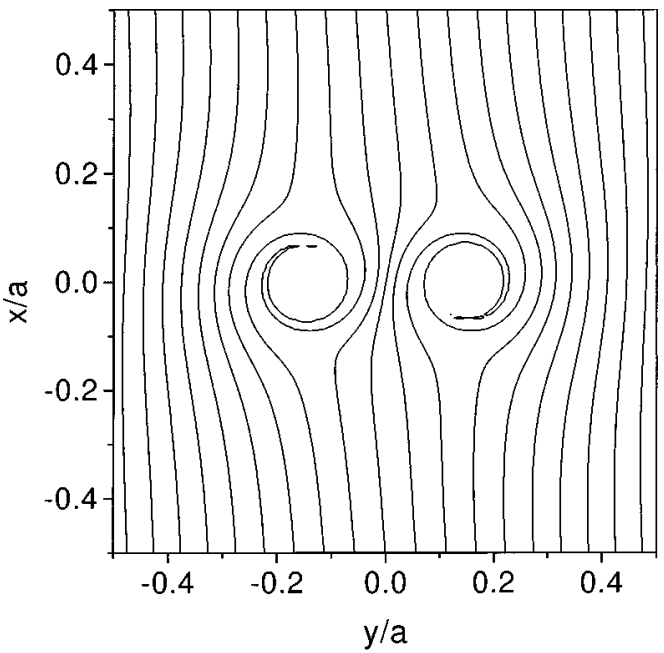

FIG. 6. Contour lines of the electrostatic potential within one unit cell of the antidot lattice described in the Appendix.

sity reaches considerably higher values between the antidots [about $3 j_{x}^{0}$ at $(x, y)=(0, a / 2)$ for a distance of $0.3 a$ between the centers of adjacent antidots, and about $1.5 j_{x}^{0}$ at $(0,0)$ for $0.7 a$. The local electron heating, which is proportional to $|\mathbf{j}|^{2}$, is even enhanced by factors of about 10 or 3 for antidot distances of $0.3 a$ or $0.7 a$, respectively.

For the sake of comparison, we show in Fig. 7(b) similar results for the corresponding lattice of antidots with equal spacings along the line at $x=0$. As expected, the peak values of $j_{x}(x, y)$ and thus of the heat production remain considerably below those of Fig. 7(a).

These results support the physical picture underlying our discussion of the breakdown currents in periodic and aperiodic antidot arrays. Details of the numerical results, e.g., the

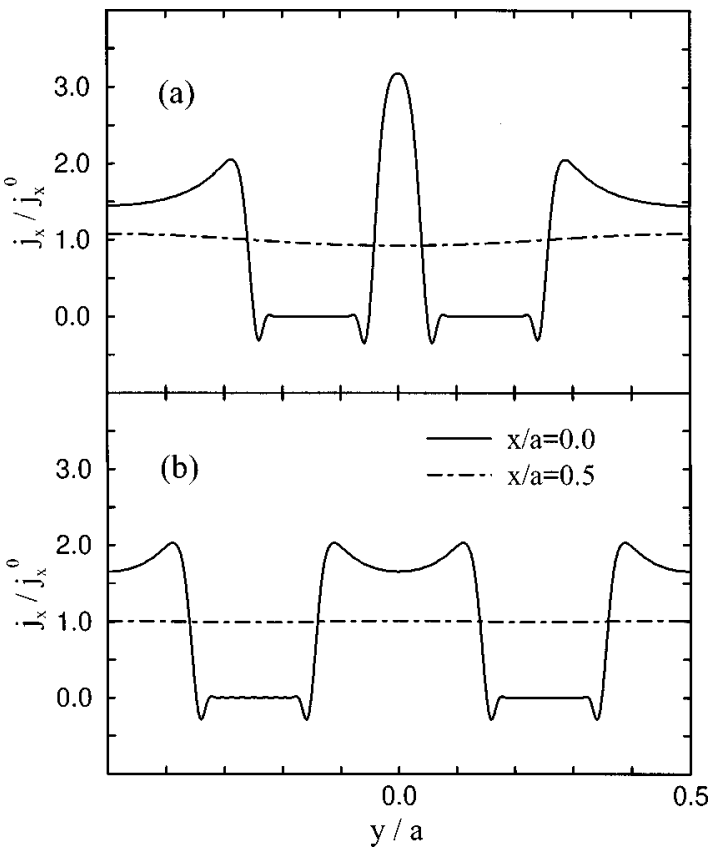

FIG. 7. Current density $j_{x}(x, y)$, in units of its average value $j_{x}^{0}$ over the unit cell, along the lines $x=0$ (solid lines) and $x=a / 2$ (dash-dotted lines) for the antidot lattice described in the Appendix with antidot parameters $R / a=0.05$, and (a) $y_{0} / a=0.15$ and (b) $y_{0} / a=0.25$.

magnitude of the backflow currents around the antidots leading to negative values of $j_{x}$, should depend on the details of our model, but not the general trends. We have confirmed this expectation by calculations with a more realistic ansatz for $\sigma_{x x}$ as a function of the local electron density (with a maximum of $\sigma_{x x}$ at half integer filling factors).
*Present address: Universität Regensburg, NWF II-Physik, Universitätsstr. 31, D-9340 Regensburg, Germany.

${ }^{1}$ K. von Klitzing, G. Dorda, and M. Pepper, Phys. Rev. Lett. 45, 494 (1980).

${ }^{2}$ S. Kawaji, H. Hirakawa, M. Nagata, T. Okamoto, T. Fukase, and T. Goto, Surf. Sci. 305, 161 (1994).

${ }^{3}$ A. Boisen, P. Bøggild, A. Kristensen, and P. E. Lindelof, Phys. Rev. B 50, 1957 (1994).

${ }^{4}$ N. Q. Balaban, U. Meirav, H. Shtrikman, and Y. Levinson, Phys. Rev. Lett. 71, 1443 (1993).

${ }^{5}$ M. E. Cage, R. F. Dziuba, B. F. Field, E. R. Williams, S. M. Girvin, A. C. Gossard, D. C. Tsui, and R. J. Wagner, Phys. Rev. Lett. 51, 1374 (1983).

${ }^{6}$ L. Bliek, E. Braun, G. Hein, V. Kose, J. Niemeyer, G. Weimann, and W. Schlapp, Semicond. Sci. Technol. 1, 110 (1986).

${ }^{7}$ Ch. Simon, B. B. Goldberg, F. F. Fang, M. K. Thomas, and S. Wright, Phys. Rev. B 33, 1190 (1986).

${ }^{8}$ P. Středa and K. von Klitzing, J. Phys. C 17, L483 (1984)

${ }^{9}$ L. Eaves and F. W. Sheard, Semicond. Sci. Technol. 1, 346 (1986).

${ }^{10}$ G. Ebert, K. von Klitzing, K. Ploog, and G. Weimann, J. Phys. C 16, 5441 (1983).

${ }^{11}$ S. Komiyama, T. Takamasu, S. Hiyamizu, and S. Sasa, Solid State Commun. 54, 479 (1985); T. Takamasu, S. Komiyama, S. Hiyamizu, and S. Sasa, Surf. Sci. 170, 202 (1986).
${ }^{12}$ S. Komiyama, Y. Kawaguchi, T. Osada, and Y. Shiraki, Phys. Rev. Lett. 77, 558 (1996).

${ }^{13}$ G. Nachtwei, G. Lütjering, D. Weiss, Z. H. Liu, K. von Klitzing, and C. T. Foxon, Phys. Rev. B 55, 6731 (1997).

${ }^{14}$ V. G. Burnett, A. L. Efros, and F. G. Pikus, Phys. Rev. B 48, 14365 (1993)

${ }^{15}$ D. Weiss, M. L. Roukes, A. Menschig, P. Grambow, K. von Klitzing, and G. Weimann, Phys. Rev. Lett. 66, 2790 (1991).

${ }^{16}$ E. Stahl, D. Weiss, G. Weimann, K. von Klitzing, and K. Ploog, J. Phys. C 18, L738 (1985).

${ }^{17}$ R. R. Gerhardts and V. Gudmundsson, Phys. Rev. B 34, 999 (1986).

${ }^{18}$ D. G. Polyakov and B. I. Shklovskii, Phys. Rev. Lett. 74, 150 (1995).

${ }^{19}$ P. Svoboda, G. Nachtwei, C. Breitlow, S. Heide, and M. Cukr, Semicond. Sci. Technol. 12, 264 (1997).

${ }^{20}$ Earlier experiments by Clark [R. G. Clark, Phys. Scr. 39, 45 (1991)] yielded a prefactor of $\sigma_{0}=e^{2} / h$. We adopted the prefactor of Refs. 18 and 19, because our sample mobilities are even higher than the values reported in Ref. 19. The latter results were well explained by long-range scattering, which is typical for high-mobility samples.

${ }^{21}$ Y. Ono, J. Phys. Soc. Jpn. 51, 237 (1982).

${ }^{22}$ D. G. Polyakov and B. I. Shklovskii, Phys. Rev. B 48, 11167 (1993). 